\title{
QUADRATIC APPROXIMATION OF GENERALIZED TRIBONACCI SEQUENCES
}

\author{
Gamaliel Cerda-Morales \\ Instituto de Matemáticas \\ Pontificia Universidad Católica de Valparaíso \\ Blanco Viel 596, Cerro Barón, Valparaíso, Chile \\ e-mail: gamaliel.cerda.m@mail.pucv.cl
}

\begin{abstract}
In this paper, we give quadratic approximation of generalized Tribonacci sequence $\left\{V_{n}\right\}_{n \geq 0}$ defined by $V_{n}=r V_{n-1}+s V_{n-2}+t V_{n-3}(n \geq 3)$ and use this result to give the matrix form of the $n$-th power of a companion matrix of $\left\{V_{n}\right\}_{n \geq 0}$. Then we re-prove the cubic identity or Cassini-type formula for $\left\{V_{n}\right\}_{n \geq 0}$ and the Binet's formula of the generalized Tribonacci quaternions.

Keywords: Binet's formula, companion matrix, generalized Tribonacci sequence, Narayana number, Padovan number, quadratic approximation, Tribonacci number.
\end{abstract}

2010 Mathematics Subject Classification: Primary: 11B39; Secondary: $15 \mathrm{~A} 24,40 \mathrm{C} 05$.

\section{REFERENCES}

[1] G. Cerda-Morales, On a Generalization of Tribonacci Quaternions, Mediterranean J. Math. 14:239 (2017) 1-12. doi:10.1007/s00009-017-1042-3

[2] M. Elia, Derived sequences, the Tribonacci recurrence and cubic forms, The Fibonacci Quarterly 39 (2001) 107-109.

[3] M. Feinberg, Fibonacci-Tribonacci, The Fibonacci Quarterly 1 (1963) 71-74.

[4] W. Gerdes, Generalized Tribonacci numbers and their convergent sequences, The Fibonacci Quarterly 16 (1978) 269-275.

[5] E. Kiliç, Tribonacci sequences with certain indices and their sums, Ars Comb. 86 (2008) 13-22.

[6] T. Koshy, Fibonacci and Lucas Numbers With Applications (John Wiley \& Sons, INC, 2001). 
[7] K. Kuhapatanakul and L. Sukruan, The generalized Tribonacci numbers with negative subscripts, Integers 14, Paper A32, 6 p. (2014).

[8] S. Pethe, Some identities for Tribonacci sequences, The Fibonacci Quarterly 26 (1988) 144-151.

[9] J.L. Ramírez and V.F. Sirvent, A note on the k-Narayana sequence, Ann. Math. Inform. 45 (2015) 91-105.

[10] A.G. Shannon and A.F. Horadam, Some properties of third-order recurrence relations, The Fibonacci Quarterly 10 (1972) 135-146.

[11] W.R. Spickerman, Binet's formula for the Tribonacci numbers, The Fibonacci Quarterly 20 (1982) 118-120.

[12] M.E. Waddill and L. Sacks, Another generalized Fibonacci sequence, The Fibonacci Quarterly 5 (1967) 209-222.

[13] C.C. Yalavigi, Properties of Tribonacci numbers, The Fibonacci Quarterly 10 (1972) 231-246.

Received 25 June 2018

Revised 23 July 2018

Accepted 3 September 2018 